\title{
El piano complementario en el conservatorio profesional de música: análisis y
}

\author{
aportaciones
}

\section{The complementary piano at the Conservatory of Music : analysis and contributions}

\author{
Isabel Romero Tabeayo*, Francisco César Rosa Napal** \\ *CMUS Profesional da Coruña, **Universidade da Coruña
}

\begin{abstract}
Resumen
Las necesidades formativas actuales en el ámbito profesional de las disciplinas instrumentales no solo entienden la actividad musical a través de la enseñanza de la interpretación. El área de piano complementario se configura como un espacio único para la experimentación musical desde la integración y práctica de los elementos constitutivos del lenguaje. El presente trabajo desarrollado dentro del marco de la investigación cualitativa, pretende detallar, analizar y comprender los principios básicos del área de piano complementario que deben ser aprendidos y transmitidos, así como, indagar sobre las concepciones, experiencias y vivencias del alumnado en el conservatorio profesional de música. Palabras clave: enseñanza musical específica, conservatorio, piano complementario, profesionalización musical.
\end{abstract}

\begin{abstract}
The current training needs in the professional field of instrumental disciplines not only understand the music business through teaching interpretation. Complementary piano area is configured as a single space for musical experimentation from the integration and practice of the constituent elements of language. This work developed within the framework of qualitative research , intended to detail, analyze and understand the basic principles of complementary piano area that must be learned and transmitted as well as inquire about the concepts, experiences and experiences of students in the professional conservatory of music.

Keywords: specific musical education, conservatory, complementary piano, musical professionalism.
\end{abstract}

\section{Justificación y contexto: necesidades y desafíos de la educación musical en el conservatorio.}

El conocimiento El conocimiento de lo artístico forma parte de los procesos de transición permanente que definen y caracterizan la peculiaridad del tiempo presente. La reflexión y el pensamiento sobre las áreas artísticas obedecen a los cambios de escenario propiciados por las nuevas tecnologías de la comunicación y el mundo de la información. Gimeno (2001), ante este tejido de interdependencia, alude a una red de intercambios, préstamos y acuerdos de cooperación que inciden en las pautas de comportamiento y los modelos culturales donde algunos de sus rasgos son conocidos e influidos entre sí.

Giráldez (2009), señala un cambio en las prácticas artísticas en relación a sus propios límites y los materiales que estas involucran. Por otra parte, reflexiona sobre el hecho del objeto artístico actual, la naturaleza de la representación y el papel del espectador o autor indicando una ruptura entre el concepto de lo estético, de lo formal y lo conceptual que nos sitúa en un nuevo y complejo marco epistemológico.

La educación artística, como disciplina que se enseña y se aprende no permanece ajena a dicha transformación y nos exige concretar lo educativo en lo artístico y lo artístico en lo educativo. Touriñan (2011) incide en la relevancia de situar y definir el arte como ámbito educativo que nos permita entender la relación artes-educación a través de sus diferentes contextos y objetivos. Distingue, por lo tanto: artes como ámbito general de educación que aportan valores vinculados al carácter y sentido propios del significado de educación; artes como ámbito de educación general, es decir, como ámbito que forma parte de la educación común de los escolares y desarrolla el sentido estético y de lo artístico, y, las artes como ámbito de desarrollo profesional y vocacional.

La relación artes- educación se configura como proceso activo, dinámico y variable. En este panorama, donde situamos la enseñanza musical impartida por los conservatorios, nos preguntamos por lo que significa ser profesional del ámbito musical en la actualidad, por la realidad a la cual nos enfrentamos como institución, y, por las necesidades formativas que de ello derivan.

Tal y como establece Sierra (2010b), la pedagogía musical no puede estar aislada, sino que debe formar parte de una concepción educativa más amplia. Igualmente, al referirnos al conservatorio como principal institución representativa de dicha profesionalización, nos cuestionamos sus objetivos reflejados en su currículum educativo así como la idoneidad y suficiencia de los mismos ante la demanda actual.

En el caso de las enseñanzas profesionales de música impartidas en el conservatorio las principales 
necesidades formativas se manifiestan como una problemática que va más allá de la interpretación musical. Desde la materia denominada piano complementario, piano funcional o piano general -dirigida a alumnos no pianistas- se establece una nueva conceptualización del instrumento, una nueva relación con el alumnado y una consecuente adaptación de objetivos, contenidos y métodos de trabajo generando un modelo educativo novedoso dentro del conservatorio.

Nos encontramos, por tanto, ante un proceso formativo que se encuentra íntimamente vinculado al concepto de aprendizaje creativo y producción creativa. Mientras las disciplinas instrumentales se ocupan, dentro del aprendizaje musical, de la formación exclusiva del repertorio solista, el área de piano complementario se configura como un espacio único para la experimentación e implantación de conceptos de aprendizaje que no son posibles de asumir por dichas especialidades.

\section{Diseño de la investigación}

\section{Objetivos de investigación}

El propósito fundamental de la investigación ha sido: comprender los principios básicos del área de piano complementario como recurso imprescindible en la formación profesional de músicos en el conservatorio. Concretamos nuestra investigación procurando:

- Indagar sobre las concepciones, experiencias y vivencias del alumnado en el conservatorio profesional de música.

- Analizar el rol que desempeña la materia de piano complementario en relación a la institución y el diseño curricular.

- Definir los elementos que sitúan al área de piano de complementario como herramienta para el desarrollo profesional de la música.

\section{Metodología de investigación}

El propósito fundamental de la investigación ha sido: comprender los principios Situamos el presente trabajo de investigación dentro del marco de la perspectiva interpretativa. Desde la metodología cualitativa nos proponemos estudiar los diferentes actores y situaciones desde su propia realidad. Pretendemos indagar sobre el significado que le atribuye a cada circunstancia y el modo en que enfatiza las relaciones y asuntos derivados.

Tal y como indica Stake (1998), el objetivo de nuestro modelo de investigación, supone comprender la complejidad y significados de la experiencia humana defendiendo la ilustración y comprensión de la realidad, el modo en que se percibe y su forma de dar respuestas. De este modo, el paradigma interpretativo, nos obliga a profundizar en cada fenómeno para obtendremos la información necesaria para analizar la particularidad de los hechos (Simons, 2010).

En este caso, diseñamos nuestra investigación a través de una entrevista en grupo. Reunido un grupo de cinco estudiantes del grado profesional del
Conservatorio Profesional de Música de A Coruña, con una clara determinación profesional pretendemos conocer y estudiar las particularidades del área de piano complementario dentro de su contexto real.

\section{Técnicas e instrumentos de investigación}

Las técnicas e instrumentos empleados para la recogida de datos de nuestra investigación han sido la entrevista en grupo y la observación participante en determinadas clases propias de la materia.

Mediante el grupo de discusión pretendemos la obtención controlada de un discurso por parte de un grupo de sujetos sobre las propuestas guiadas, en este caso, del investigador. Igualmente, nos planteamos conocer las preocupaciones, sentimientos y actitudes de los participantes.

Procuramos favorecer una discusión libre y moderar la dirección del discurso hacia los temas de investigación, priorizando la visión colectiva y promoviendo la discusión entre los diferentes rasgos que definen al grupo. En este caso, realizamos dos sesiones de una hora cada una en el conservatorio de música con cinco estudiantes del grado profesional.

\section{Informe de investigación}

El propósito fundamental de la investigación ha sido: comprender los principios básicos del área de piano complementario como recurso imprescindible en la formación profesional de músicos en el conservatorio. Concretamos nuestra investigación procurando: La materia de piano complementario irrumpe en el conservatorio gallego con el Decreto 253/1993, de 29 de julio, estableciendo el currículo del grado elemental y del grado medio enmarcado en la Ley Orgánica 1/1990, de 3 de octubre, de Ordenación General del Sistema Educativo. Dirigida a estudiantes no pianistas del grado medio, se estructuraba a lo largo de los primeros cuatro cursos con una duración semanal de treinta minutos por alumno.

Posteriormente, la Ley Orgánica 2/2006, de 3 de mayo, de Educación con el Decreto 203/2007, de 27 de septiembre, establece el currículo de las enseñanzas profesionales de régimen especial de música. En esta ocasión, la asignatura de piano complementario, continúa con la misma carga lectiva y con los principios de la reforma anterior, únicamente modifica su ubicación en el grado profesional; corresponde a los cuatro últimos cursos del mismo.

El piano complementario, en el panorama internacional, presenta múltiples concepciones sobre su finalidad y lo que debe presentar al alumnado. Los sistemas más tradicionales asumen su principal función en el trabajo exclusivo del repertorio pianístico, sin embargo, las nuevas tendencias lo vinculan a la creatividad, la flexibilidad y la exploración de nuevos campos que ayuden a los alumnos y alumnas a comprender el papel social, cultural y artístico del músico. 
En este caso es pertinente identificar unidades de aprendizaje que justifiquen una propuesta y diseño metodológico coherentes que den respuesta a lo inconexo de la praxis artístico-musical presente. Siguiendo a Gainza (2011), entendemos el momento actual como una oportunidad para revitalizar la pedagogía de la música y comprometida con las necesidades y circunstancias existentes.

A partir del análisis de los datos obtenidos, centramos nuestra atención en los siguientes aspectos tal y como refleja la Figura 1.

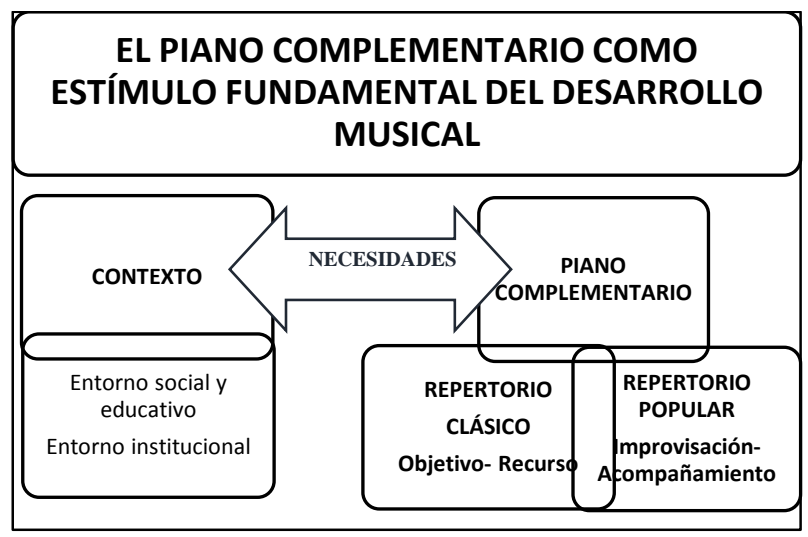

Figura 1. Posibilidades formativas del piano complementario.

El piano como herramienta para el desarrollo auditivo y la integración del lenguaje musical: el acompañamiento pianístico

El propósito fundamental de la investigación ha sido: comprender los principios Las disciplinas artísticas sitúan su aprendizaje bajo los principios de la práctica. La información requerida desde el piano contribuye a la ejecución de estructuras lingüísticas comprensibles que respondan a las necesidades de la interpretación. De esta manera, nos encontramos con las siguientes afirmaciones: "Nos ofrece el desarrollo armónico" (Estudiante 2, E1.2), "La diferenciación de manos ayuda a distinguir planos... es fundamental el pensamiento polifónico porque soy más consciente de lo que escucho”. (Estudiante 1, E1.2).

Tal y como afirma Gordon (1997), la audición o habilidad para escuchar y entender la música es necesaria para la descodificación de dicho sistema lingüístico; tocar, leer, pensar estructuras musicales. Lo importante de este hecho radica en la compartimentación de contenidos así como de su aislamiento, nos encontramos ante una desestructuración de las materias y del sistema educativo en general. Tal y como recogemos en nuestra entrevista: “...en armonía no tocamos los ejercicios... el piano se abre ante ti. Trabajas la música y puedes ser más analítico... luego lo puedes aplicar en tu propio instrumento” (Estudiante 4, E1.2); “...la materia está muy estructurada, primero practicamos una pequeña cosa y después la aplicamos en el conjunto... “(Estudiante 5, E1.5).
Elliott (2009), señala que escuchar no es el equivalente auditivo de un proceso de copia, al contrario, escuchar es un proceso de construcción cognitivaafectiva (Figura 2). Mediante la escucha se establecen las principales relaciones motoras entre la ejecución y el producto sonoro final: "El piano ayuda sobre todo a tocar en una formación con piano..., ahora cuando ensayo con mi pianista comprendo me fijo más en lo que va haciendo” (Estudiante 5, E1.2).

\section{ESCUCHAR $\underset{\sim}{\longrightarrow}$ COMPRENDER $\underset{\sim}{\longrightarrow}$ EXPRESAR}

Figura 2. Proceso de musicalización

La asignatura de piano complementario se configura como una materia de conocimiento muy amplia, las posibilidades que nos ofrece son múltiples y heterogéneas. En este sentido destacamos la adecuación e incursión en la música popular, la cual, se convierte en uno de los recursos más eficaces para la desinhibición musical, la creatividad y la espontaneidad: "lo bueno es no sólo ceñirse a la música clásica” (Estudiante 1, E1.5).

Considerando el trabajo en el aula como la interacción de los elementos constitutivos del lenguaje, una de las principales responsabilidades de la materia radica en desarrollar las habilidades propias del acompañamiento pianístico de diferentes textos y melodías (ya sean de carácter clásico o popular).

Entendemos, por lo tanto, que el empleo del acompañamiento pianístico en la formación musical se configura como un elemento observable susceptible de ser aprendido y como guía, representación o ejemplificación de conceptos imitables. Incluso, en este sentido el alumnado es consciente de la necesidad de una preparación en este sentido: “yo necesito saber acompañar para poder hacerlo con mis alumnos en un futuro" (Estudiante 4, E1.5).

Partiendo de la práctica vocal nos proponemos analizar, concretar y expresar la idea musical que hemos de acompañar: “...si no lo sabes cantar, no lo sabes tocar” (Estudiante 4, E1.6); "incluso, la forma en cómo lo cantas es importante, tiene que ser expresivo, necesita de fraseo" (Estudiante 3, E1.6)

La condición imprescindible para la elaboración de un acompañamiento radica en los siguientes aspectos:

- Conocimiento de los elementos constitutivos de aquello que se ha de acompañar (ritmo, melodía, armonía, forma, estilo).

- Adecuación del acompañamiento pianístico a los objetivos didácticos previamente definidos.

- Control sobre el teclado para establecer un modelo correcto sobre la idea musical a ejemplificar.

La clase colectiva como recurso en la formación de la personalidad artística

El propósito fundamental de la investigación ha sido: comprender los principios Las especialidades 
instrumentales, ligadas al estudio del repertorio solista, se han impartido, tradicionalmente, dentro del modelo de clase individual. No obstante, la experiencia de la clase colectiva comienza a valorarse como recurso necesario en la formación musical por las posibilidades didácticas que nos ofrece: “...En la clase grupal el resto de compañeros te escuchan y pierdes el miedo a tocar” (Estudiante 2, E1.4.); "Puedes aprender de los demás" (Estudiante 5, E1.4.)

Gordon (1997), se refiere a la clase colectiva como un espacio en el que las diferencias musicales individuales pueden ser conciliadas y los estudiantes se verán obligados a aprender a adaptarse al sonido, por lo que tendrán que escuchar, y de este modo se fomenta el desarrollo auditivo.

Del mismo modo Gainza (2007), entiende la práctica en grupo como la posibilidad de compenetración rítmica, simultaneidad de ataques, equilibrio dinámico, fraseo y gesto musical. Tal y como recogemos en nuestros datos: "Estudiar en grupo te abre las relaciones y compartimos ese tiempo de trabajo" (Estudiante 4, E1.4.). Igualmente, la experiencia musical colectiva, orienta las exigencias interpretativas hacia la percepción sonora de lo que se produce de un modo natural (Gordon, 2003).

En este punto, el planteamiento del aprendizaje pianístico en grupo ha de entenderse como un recurso que en ningún caso pretende sustituir la dirección y supervisión del trabajo individual de cada alumna y alumno. Se trata de explotar las posibilidades que nos ofrece para integrar materiales fundamentados en la audición, expresión y coordinación del grupo sobre el instrumento: "La individualidad en el instrumento es importante, pero la clase colectiva es fundamental..., me gustaría que el trabajo empezara sólo de forma individual y después poder aplicarlo en grupo. A veces no estoy del todo seguro en grupo y necesito más atención...” (Estudiante 1, E1.4).

\section{Conclusiones}

La educación artística cada vez asume un mayor reconocimiento dentro de las ciencias sociales. Ante una emergente demanda que requiere espacios para la reflexión y la comprensión de las nuevas prácticas en educación musical surge la necesidad de establecer unos principios sólidos que a partir de la investigación generen modelos dedicados a cómo aprender.

En el contexto definido por las enseñanzas profesionales de música en el conservatorio, las metodologías tradicionales siguen siendo válidas, pero, es una realidad que muchas de ellas no respondan a las necesidades actuales. A ello debemos añadirle la complejidad que supone el diseño curricular de las mismas, y lo urgente de asumir una verdadera interdisciplinariedad.

En la medida en que se logran metas musicales desde el afecto, el conocimiento y la interpretación, se inicia un proceso de retroalimentación que va perfilando los principios propios de la identidad profesional generando un impulso motivador o vocacional por lo que es prioritario concretar y determinar las estrategias didácticas adecuada que deben proporcionar al alumno motivación, información y orientación para alcanzar los objetivos previstos.

\section{Referencias}

Elliot, D. (2009): “La comprensión musical, las obras musicales y la expresión de los sentimientos: Implicaciones para la educación”. En Lines, D. K. (Comp.) (2009): La educación musical para el nuevo milenio. Madrid. Morata. Pp 123- 136.

Furlan, A. (1991). Metodología de la enseñanza. En Aportaciones a la Educación Superior. Mexico. UNAM, México. 1989. pp. 61-83.

Gainza, V.H de (2011). “Educación musical siglo XXI: problemáticas contemporáneas”. Revista da ABEM, vol.19, n.25. Pp 11-18.

Gainza, V.H. de (2007). Didáctica del piano. Piano Joven. Barcelona: Dinsic.

Gimeno Sacristán, J. (2001): “El significado y la función de la educación en la sociedad y cultura globalizadas”, en Revista de Educación, número extraordinario 2001, 121-142.

Giráldez, A. (2009): "Fundamentos metodológicos de la educación artística”. en Jiménez (Coord) (2009): Educación artística, cultura y ciudadanía. Madrid. Metas Educativas 2021. Fundación Santillana.

Gordon, E. (1997). La clase colectiva de instrumento. Eufonía nº7, pp. 91-100.

Gordon, S. (2003). Técnicas maestras de piano.

Barcelona: Ma Non Troppo.

Lines, D. K. (2009). La educación musical para el nuevo milenio. Madrid. Morata.

Sierra, J.A. (2010b): "Formar profesionales de música”, en Scherzo n²52, pp. 148-150.

Simons, H. (2010): El estudio de caso: teoría y práctica. Madrid, Morata

Stake. R. E. (1998). Investigación con estudios de caso. Madrid. Morata.

Touriñán, J. (2011). Claves para aproximarse a la educación artística en el sistema educativo: educación "por" las artes y educación "para" un arte. Estudios sobre educación, vol.21. pp. 61-81. Universidad de Navarra. 